\title{
Extraction sélective des actinides : application à l'analyse radiotoxicologique
}

\author{
C. DINSE*, C. COSSONNET*, N. BAGLAN*, Z. ASFARI**, J. VICENS**
}

(Manuscrit reçu le 17 mars 1997, révisé le 15 juillet 1997, accepté le 30 août 1997)

RÉSUMÉ Le rendement d'extraction de l'uranium a été étudié par extraction liquideliquide, à l'aide d'une molécule macrocyclique, le calix[6]arène, $\mathbf{4}_{6}$, fonctionnalisé par des groupements acide carboxylique. La phase aqueuse comprenait l'uranium en présence d'ions sodium et la phase organique le calixarène dissous dans le benzène. L'étude des paramètres d'extraction a permis de proposer un mécanisme d'extraction. Le rendement d'extraction augmente avec le pH et atteint un maximum voisin de $100 \%$ pour un $\mathrm{pH}>3,7$. Sur des solutions contenant en plus de l'uranium des ions sodium, marquées au ${ }^{22} \mathrm{Na}$ et/ou du plutonium 239 , l'extraction de l'uranium reste optimale. Sur des échantillons d'urine, après minéralisation, environ $100 \%$ de l'uranium sont extraits par le calixarène. Ces premiers résultats montrent que le calixarène choisi présente une structure adéquate vis-à-vis de la structure de coordination de l'ion uranyle, permettant ainsi une extraction sélective de l'uranium.

ABSTRACT Selective extraction of actinides: Application to radiotoxicological analysis. The extraction efficiency of uranium has been studied in a two phases solventextraction system with a macrocyclic molecule, a calix[6]arene, bearing carboxy groups. The aqueous phase contained uranium in presence of sodium ions and the calixarene was dissolved in benzene. The study of the extraction parameters allowed to propose an extraction mechanism. The extraction efficiency was found to increase with $\mathrm{pH}$, with an optimal value around $100 \%$. On synthetic solutions containing in addition to uranium sodium ions (spiked with ${ }^{22} \mathrm{Na}$ ) and/ or ${ }^{239} \mathrm{Pu}$, no decrease of uranium extraction was observed. When applied to urine samples, after organic digestion, about $100 \%$ of uranium have been extracted by the calixarene at $\mathrm{pH}>3.7$. These first results have shown that the calixarene which has been selected has an adequate structure relative to the uranyl ion co-ordination structure, which permits a selective extraction of uranium.

\footnotetext{
Institut de Protection et de Sûreté Nucléaire, département de la santé de l'homme et de dosimétrie, service de dosimétrie, IPSN, B.P. ${ }^{\circ}$ 6, 92265 Fontenay-aux-Roses Cedex, France.

** École européenne chimie polymères matériaux de Strasbourg, ECPM, URA405 CNRS, 1, rue Blaise Pascal, 67008 Strasbourg Cedex, France.
} 


\section{Introduction}

\subsection{Les calixarènes récepteurs et transporteurs de cations}

La chimie de coordination des ligands macrocycliques suscite un grand intérêt dans des domaines très variés (Alexander, 1995) parmi lesquels la catalyse, la synthèse d'antibiotiques, ou encore la mise au point d'agents thérapeutiques pour le traitement d'intoxication par les métaux. Bien que de nombreux travaux aient été menés dans le domaine de la complexation des cations, la recherche de nouveaux ligands est nécessaire pour parvenir à augmenter la sélectivité et l'efficacité d'extraction, en particulier dans le cadre de cette étude pour les milieux biologiques qui concernent l'analyse radiotoxicologique.

Jusque dans les années soixante, très peu de ligands capables de complexer les cations alcalins étaient connus (Vicens et Böhmer, 1991). C'est l'étude des mécanismes de transmission de l'influx nerveux qui a permis de mettre en évidence la structure des récepteurs du potassium. Des études cristallographiques ont montré l'inclusion du cation dans une cavité délimitée par une répartition cyclique d'atomes d'oxygène. À la suite de ces travaux, les premiers ionophores de synthèse ont été mis au point. Pedersen a réalisé la synthèse de polyéthers macrocycliques (Vicens et Böhmer, 1991) caractérisés par la présence d'une cavité centrale hydrophile : les éther-couronnes ou coronands, (Fig. 1). Une amélioration significative de la sélectivité de complexation a été apportée par les cryptands développés par le professeur Lehn (Dietrich, 1991). Les cryptands (Fig. 1) diffèrent des coronands par leur nature bicyclique. Ils forment une cavité sphérique tridimensionnelle capable d'envelopper complètement le cation complexé.

Toutes ces familles de ligands diffèrent par leur degré de préorganisation, ce qui se traduit pour les complexes par des différences de stabilité thermodynamique et cinétique. Cram a clarifié le rôle important de la préorganisation et de la complémentarité dans la détermination de la stabilité des complexes (Vicens et Böhmer, 1991). Le principe de préorganisation a pour énoncé : " plus les modifications dans l'organisation du récepteur, du substrat et du solvant, nécessaires pour la complexation, sont faibles, plus la stabilité du complexe formé est forte ». La reconnaissance structurale entre le récepteur et le substrat est contrôlée par le principe de complémentarité, ce qui signifie que "pour complexer le substrat, le récepteur doit posséder des sites de coordination qui en même temps doivent entrer en contact et attirer les sites de coordination du substrat, sans générer de répulsion importante sur les sites libres».

La considération de ces principes a conduit à l'utilisation des calixarènes (Fig. 1) en tant que macrocycles « d'organisation ordonnée ». Les calixarènes ont en effet une structure intéressante pour la complexation des cations mais ils ont surtout la propriété de pouvoir être fonctionnalisés de façon très variée. 
<smiles>c1ccc2c(c1)OCCOCCOc1ccccc1OCCOCCO2</smiles>

I Coronand

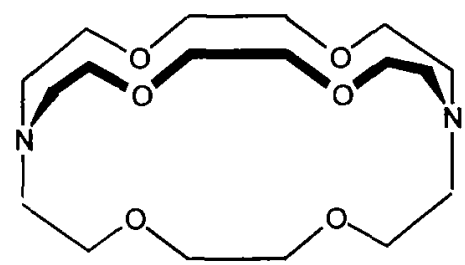

II Cryptand

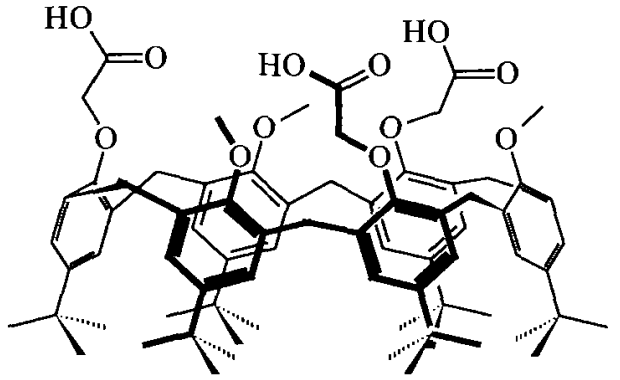

III Calix[6]arène-triacide

Fig. 1. - Differentes molécules macrocycles.

Various macrocyclic molecules.

Les calixarènes (Gutsche, 1989) sont des macrocycles formés de $n$ unités phénoliques reliées entre elles par des ponts méthyléniques situés en position ortho des groupes hydroxyles. Le terme de calixarène a été introduit en 1987 par Gutsche afin de simplifier la nomenclature de ces macrocycles. Ce nom provient du grec calix signifiant vase ou calice et arène qui indique la présence de noyaux aromatiques formant un cycle. Le nombre de motifs phénoliques constituant la molécule est indiqué en intercalant le chiffre correspondant entre calix et arène. La nature et la position des substituants présents sur les noyaux aromatiques sont précisées avant le terme calix[n]arène.

Les calixarènes offrent différentes possibilités de modifications chimiques au niveau de la position en para du noyau aromatique (upper rim), au niveau des groupements méthylènes, et surtout au niveau des fonctions phénoliques (lower rim). Ces molécules peuvent être solubles, soit en phase aqueuse, soit en phase organique, par fonctionnalisation particulière en para des fonctions hydroxyles. Il est également possible de greffer à la place des fonctions hydroxyles des fonctions ayant une affinité plus spécifique au cation que l'on désire complexer.

Les calixarènes offrent ainsi la possibilité de jouer sur plusieurs paramètres, à savoir, la taille de la cavité, le greffage de fonctions et leurs propriétés de solubilité, afin de construire la molécule adéquate vis-à-vis de l'élément à extraire. 


\subsection{Application à l'analyse radiotoxicologique}

Les propriétés des calixarènes sont particulièrement intéressantes lorsqu'il faut extraire un cation (l'uranium par exemple) d'un milieu complexe, tel que l'urine, où se trouvent un grand nombre d'autres cations et anions. La présente étude concerne l'extraction de l'uranium dans l'urine par un calixarène. Les premiers résultats obtenus (Baglan et al., soumis à publication) sont résumés dans ce document.

Le dosage de l'uranium dans l'urine est effectué en routine pour la surveillance des travailleurs de l'industrie nucléaire soumis à des risques de contamination interne. La technique utilisée habituellement pour la détection des actinides est la spectrométrie alpha. Une bonne détection implique la réalisation de sources en couche mince, donc la séparation sélective de l'uranium des autres impuretés et des autres actinides éventuellement présents dans l'urine. De nombreux autres cations métalliques étant présents en quantité macroscopique dans les urines, il est nécessaire d'utiliser un ligand très spécifique de l'uranium.

Pour déterminer le calixarène le plus adapté à l'extraction sélective de l'ion uranyle $\mathrm{UO}_{2}^{2+}$, les propriétés des complexes de l'ion uranyle ont été exploitées. Des études cristallographiques par diffraction des rayons X (Araki et al., 1993) ont établi que les complexes de l'ion uranyle adoptent une structure pseudoplanaire hexacoordonnée, ce qui n'est pas le cas des autres cations métalliques. Ceci suggère l'utilisation d'un macrocycle hôte ayant un arrangement coplanaire de six groupes ligands spécifiques pour l'ion uranyle (Shinkai et al., 1986,1987). Toutefois, des travaux concernant le calix[6]arène substitué par six fonctions acide carboxylique ont montré une diminution de la sélectivité lorsque $\mathrm{UO}_{2}^{2+}$ se trouve en compétition avec d'autres cations métalliques lors de l'extraction (Nagaski et Shinkai, 1991). En réalité, seuls trois groupements acide carboxylique suffisent pour complexer l'uranium en milieu alcalin, les trois autres étant responsables de la diminution de la sélectivité. Le calix[6]arène choisi possède donc trois fonctions méthyles en remplacement des trois fonctions acide carboxylique superflues. Cette molécule a été sélectionnée de façon à imiter la structure du complexe stable $\mathrm{UO}_{2}\left(\mathrm{CO}_{3}\right)_{3}^{4-}$. L'arrangement des groupes carboxylates confère à la molécule une symétrie de type $\mathrm{C}_{3}$ permettant d'obtenir la structure hexacoordonnée requise.

\section{Matériels et méthodes}

\subsection{Matériels}

Le composé $46: 5,11,17,23,29,35$-hexa-tert-butyl-37, 39, 41-tri(carboxyméthy1)-38, 40, 42-trimethoxy-calix[6]arène a été synthétisé (Fig. 2) à partir du produit $1_{6}$ par l'intermédiaire des composés $2_{6}$ et $3_{6}$. Cette synthèse décrite 
dans la littérature (Casnati et al., 1991; Otsuka et al., 1995) a été réalisée sans modifications. Le rendement global est de l'ordre de $10 \%$.

La caractérisation du composé obtenu a été réalisée à l'aide du spectre ${ }^{1} \mathrm{H}$ RMN du ligand libre dans $\mathrm{CDCl} 3$ faisant apparaître les signaux suivants : 6,98 (s, 6H, ArH meta $\mathrm{OCH}_{2} \mathrm{COOH}$ ), 6,94 (s, 6H, ArH meta $\left.\mathrm{OCH}_{3}\right), 3,90(\mathrm{~s}, 6 \mathrm{H}$ $\left.\mathrm{OCH}_{2} \mathrm{COOH}\right), 3,83$ (s, 12H ArCH $\left.\mathrm{Ar}\right), 3,73\left(\mathrm{~s}, 9 \mathrm{H}, \mathrm{OCH}_{3}\right), 1,12(\mathrm{~s}, 27 \mathrm{H}$, $\mathrm{C}_{4} \mathrm{H}_{9}$ para $\left.\mathrm{OCH}_{2} \mathrm{COOH}\right), 1,09\left(\mathrm{~s}, 27 \mathrm{H}, \mathrm{C}_{4} \mathrm{H}_{9}\right.$ para $\left.\mathrm{OCH}_{3}\right)$.

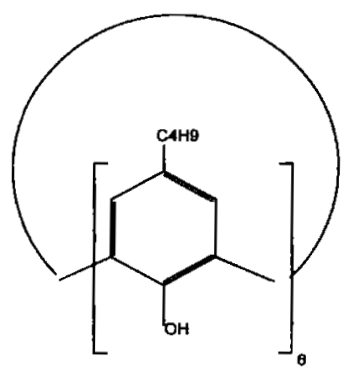

$1_{6}$

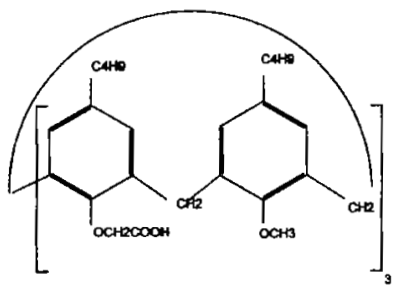

$4_{6}$

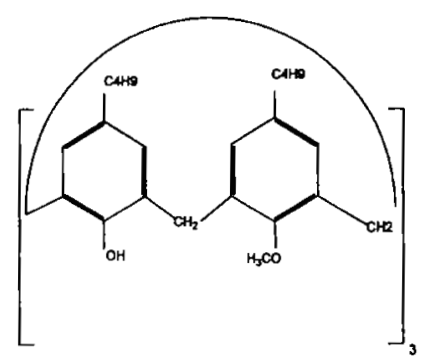

26

$\mathrm{BrCH}_{2} \mathrm{COOEt}, \mathrm{Cs}_{2} \mathrm{CO}_{3}$

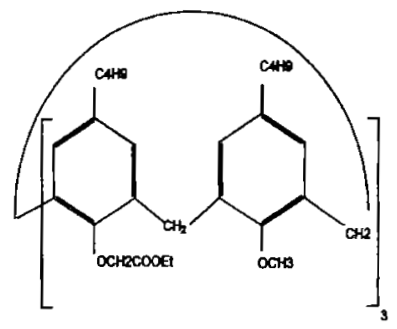

$\mathrm{KOH}, \mathrm{EtOH}, \mathrm{H}_{2} \mathrm{O}$

36

Fig. 2. - Synthèse du 5,11,17,23,29,35-hexa-tert-butyl-37,39,41-tri(carboxyméthyl)38,40,42-triméthoxy-calix[6]arène.

Synthesis of 5,11,17,23,29,35-hexa-tert-butyl-37,39,41-tri(carboxymethyl)$38,40,42$-trimethoxy-calix[6]arene. 


\subsection{Extraction par solvant}

Une solution organique (benzène lavé avec de l'acide nitrique 1 mol..$^{-1}$ ou dichlorométhane) contenant le calixarène $4_{6}$ a été mélangée avec une solution aqueuse contenant ${ }^{233} \mathrm{U}$ à $10^{-7} \mathrm{~mol} . .^{-1}\left(T_{1 / 2}=1,59 \times 10^{5}\right.$ ans, $\left.<\infty=4814 \mathrm{keV}\right)$ et du chlorure de sodium à $4 \times 10^{-2}$ mol..$^{-1}$ marqué au ${ }^{22} \mathrm{Na}\left(T_{1 / 2}=2,60\right.$ ans, $\left.E_{\gamma}=1274,5 \mathrm{keV}\right)$.

Le mélange a été placé sous agitation douce pendant deux heures à température ambiante. L'équilibre d'extraction a été atteint au bout d'environ une heure.

Les deux phases ont été séparées et les concentrations en uranium et sodium mesurées dans chaque phase. L'activité de ${ }^{233} \mathrm{U}$ a été mesurée par spectrométrie alpha sur source électrodéposée tandis que la distribution du ${ }^{22} \mathrm{Na}$ a été suivie par spectrométrie gamma.

Le $\mathrm{pH}$ de la solution aqueuse était ajusté initialement et mesuré une fois l'équilibre atteint.

\subsection{Coefficient de distribution}

Les calix[6]arènes fonctionnalisés par des groupements acide carboxylique (Montavon, sous presse) sont des macrocycles possédant des protons potentiellement échangeables.

L'extraction liquide-liquide de l'ion métallique $\left(\mathrm{UO}_{2}^{2+}\right.$ ou $\left.\mathrm{Na}^{+}\right)$par l'agent complexant, le calix[6]arène-triacide symbolisé $\mathrm{LH}_{3}$, peut donc être représentée par un échange proton-cation :

$$
a \mathrm{LH}_{3 \text { orga }}+b \mathrm{M}_{\text {aq }}^{n+} \leftrightarrow\left(\mathrm{L}_{a} \mathrm{M}_{b} \mathrm{H}_{3 a-b n}\right)_{\text {orga }}+b n \mathrm{H}_{\mathrm{aq}}^{+} .
$$

Pour tous les échantillons la force ionique étant toujours inférieure à $10^{-1}$, les coefficients d'activité sont pris égaux à 1 . Dans ces conditions, les activités et les concentrations sont équivalentes.

La constante thermodynamique d'extraction est donnée par la relation suivante :

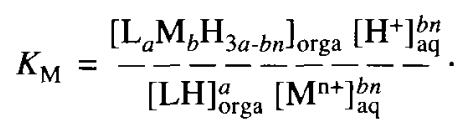

Le coefficient de distribution $D_{\mathrm{M}}$ est le rapport de la concentration en métal M de la phase organique sur celle de la phase aqueuse :

$$
D_{\mathrm{M}}=\frac{\left[\mathrm{M}_{\mathrm{total}}\right]_{\mathrm{orga}}}{\left[\overline{\mathrm{M}}_{\mathrm{total}}\right]_{\mathrm{aq}}} .
$$


En combinant les équations (1) et (2), $K_{\mathrm{M}}$ peut s'écrire :

$$
\begin{gathered}
K_{\mathrm{M}}=\frac{\left[\mathrm{M}_{\text {total }}\right]_{\text {orga }}}{\left[\mathrm{M}_{\text {total }}\right]_{\mathrm{aq}}} \\
\log D_{\mathrm{M}}=\log K_{\mathrm{M}}+a \log \left(\mathrm{LH}_{3}\right)+b n \mathrm{pH} .
\end{gathered}
$$

Le nombre de protons échangés est donné par la pente de la courbe $\log D_{\mathrm{M}}$ en fonction du $\mathrm{pH}$ (paramètre bn de l'Éq. (4)). Le nombre de molécules de ligand mis en jeu pour l'extraction est donné par la pente de la courbe $\log D_{\mathrm{M}}$ en fonction de $\log \left(\mathrm{LH}_{3}\right)$ (paramètre $a$ de l'Éq. (4)).

\section{Résultats}

Une étude préalable a été réalisée en utilisant le dichlorométhane comme solvant (Baglan et al., soumis à publication). Afin d'améliorer la solubilité du calixarène et permettre ainsi de travailler à des concentrations plus élevées, le benzène a été retenu comme solvant pour la suite de ces essais, malgré son caractère toxique. Les autres solvants aromatiques testés permettaient de dissoudre le calixarène, mais un précipité apparaissait à l'interface lors de l'extraction.

\subsection{Influence $d u \mathrm{pH}$}

La variation de $\log D_{\mathrm{U}}$ en fonction du $\mathrm{pH}$ est illustrée sur la figure 3. Dans la première partie de la courbe pour des valeurs de $\mathrm{pH}$ comprises entre 2,5 et 3,7, le pourcentage d'extraction augmente avec le $\mathrm{pH}$. Pour des valeurs de $\mathrm{pH}$ supérieures à 3,7 , un palier à $100 \%$ d'extraction est atteint.

Pour des valeurs de $\mathrm{pH}$ inférieures à 3,7 , les courbes, $\log D_{\mathrm{U}}=f(\mathrm{pH})$, à différentes concentrations en ligand, sont toutes des droites de pente égale à 2 , ce qui correspond bien à l'extraction de l'ion $\mathrm{UO}_{2}^{2+}$.

Par ailleurs dans la même gamme de $\mathrm{pH}$, l'extraction du sodium n'a jamais êté observée.

Ces résultats démontrent l'extraction sélective de $\mathrm{UO}_{2}^{2+}$ dans ces conditions opératoires.

\subsection{Stoechiométrie des espèces extraites}

La courbe $\log D_{U}$ en fonction de $\log \left(\mathrm{LH}_{3}\right)$ pour $\mathrm{pH}=2,5$ (Fig. 4) est une droite de pente égale à 1 , ce qui signifie qu'une seule molécule de ligand est mise en jeu au cours de l'extraction. 


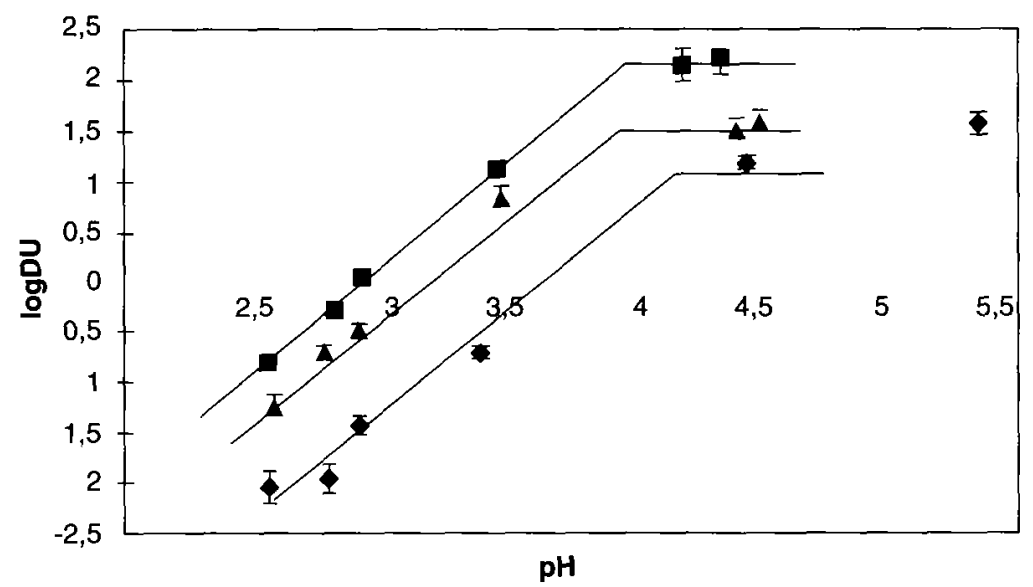

Fig. 3. - Extraction de l'uranium par $\mathrm{LH}_{3}$ dans le benzène à $293 \mathrm{~K}$, en fonction du pH pour

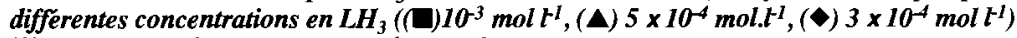
${ }^{233} \mathrm{U} 10^{-7} \mathrm{~mol} \mathrm{H}^{-1}$ et $\mathrm{NaCl} 4 \times 10^{-2}$ mol $\mathrm{H}^{-1}$ dans la phase aqueuse.

Extraction of uranium by $\mathrm{LH}_{3}$ in benzene at $293 \mathrm{~K}$, as a function of $\mathrm{pH}$ for various $\mathrm{LH}_{3}$ concentrations $(\mathbf{(}) 10^{-3} \mathrm{~mol}^{-1},(\Delta) 5 \times 10^{-4}$ mol..$^{-1}$, (४) $3 \times 10^{-4}$ mol..$\left.^{-1}\right)$ with ${ }^{233} \mathrm{U}^{10^{-7}} \mathrm{~mol} \mathrm{l}^{-1}$ and $\mathrm{NaCl} 4 \times 10^{-2} \mathrm{moll}^{-1}$ in the aqueous phase.

Dans cette gamme de $\mathrm{pH}$ et pour ces concentrations d'uranium, le diagramme de répartition des espèces de l'uranium en solution aqueuse, tracé à l'aide des données thermodynamiques (Grenthe et al, 1992) nous permet de conclure que l'ion uranyle est l'espèce prépondérante en phase aqueuse.

Deux protons étant échangés, une seule molécule de calixarène étant mise en jeu et le sodium n'étant pas extrait, le mécanisme d'extraction suivant peut être proposé :

$$
\mathrm{LH}_{3 \text { orga }}+\mathrm{UO}_{2 \mathrm{aq}}^{2+} \leftrightarrow \mathrm{LHUO}_{2 \text { orga }}+2 \mathrm{H}_{\text {aq }}^{+}
$$

\subsection{Sélectivité vis-à-vis d'autres cations}

La sélectivité du calix[6]arène-triacide $4_{6}$ vis-à-vis de l'ion uranyle a été étudiée en examinant l'influence de deux cations : le sodium et le plutonium. Le benzène a également été utilisé comme solvant.

Dans un premier temps, l'extraction du sodium a été étudiée à partir de solutions aqueuses contenant ou non des ions uranyles. Aucune extraction du sodium n'a été observée.

Dans un second temps, l'extraction du plutonium a été étudiée dans des conditions de $\mathrm{pH}$ de la phase aqueuse pour lesquelles l'extraction de l'uranium est 
optimale $(\mathrm{pH}=3,8), \mathrm{Ex}_{\mathrm{U}}=(100 \%)$. Pour vérifier la sélectivité du calixarène, plusieurs concentrations d'uranium et de plutonium ont été utilisées, dont celles correspondant à des concentrations voisines pour les deux éléments, situation extrême qui n'est pas rencontrée dans le cadre de la surveillance du travailleur.

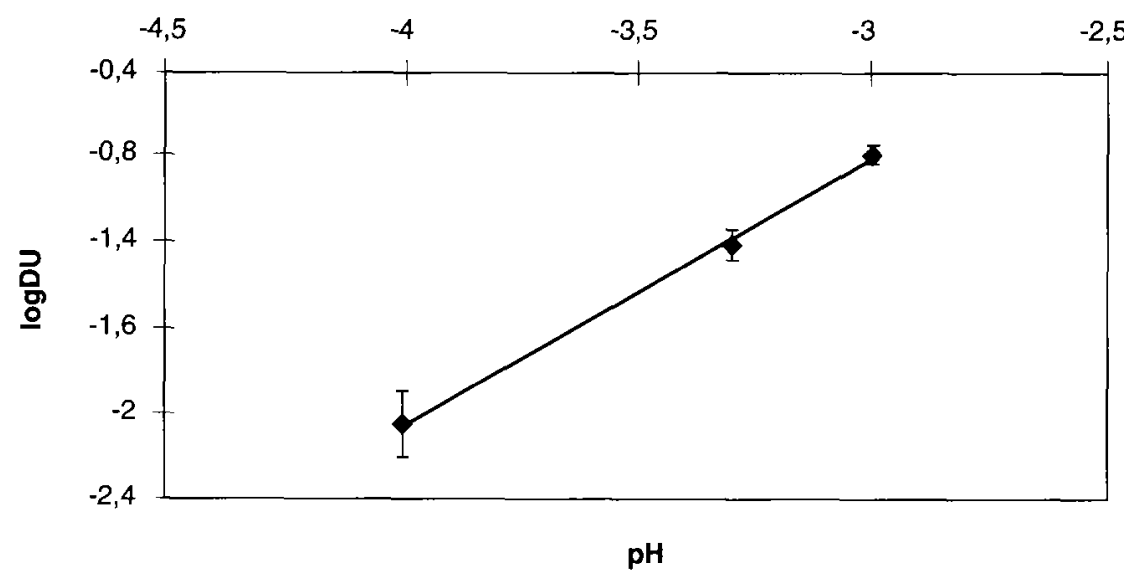

Fig. 4. - Extraction de l'uranium par $\mathrm{LH}_{3}$ dans le benzène à $293 \mathrm{~K}$, en fonction de la concentration en ligand, à pH constant $\left({ }^{233} \mathrm{U} 10^{-7}\right.$ mol $t^{-1}, \mathrm{NaCl} 4 \times 10^{-2}$ mol $l^{-1}, \mathrm{pH}=2,58$ dans la phase aqueuse).

Extraction of uranium by $\mathrm{LH}_{3}$ in benzene at $293 \mathrm{~K}$, as a function of ligand concentration at constant $\mathrm{pH}\left({ }^{233} \mathrm{U} 10^{-7} \mathrm{~mol} \mathrm{l}^{-1}, \mathrm{NaCl} 4 \times 10^{-2}\right.$ mol $\mathrm{H}^{-1}, \mathrm{pH}=2,58$ in the aqueous phase $)$.

Les résultats obtenus lors de ces premiers essais sont reportés dans le tableau I. Ils montrent que la présence d'autres cations tels que le sodium et le plutonium n'influe pas sur le pourcentage d'extraction de l'uranium.

\section{TABLEAU I}

Extraction sélective de l'uranium dans des solutions contenant du plutonium. Selective extraction of uranium in solutions containing plutonium.

\begin{tabular}{|c|c|c|}
\hline Compostion de li phate & Pourceptage d'extraction & 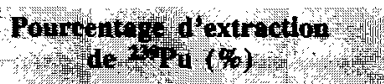 \\
\hline $\begin{array}{c}{\left[{ }^{233} \mathrm{U}\right]=10^{-7} \mathrm{~mol} \mathrm{l}^{-1}} \\
{\left[{ }^{239} \mathrm{Pu}\right]=10^{-8} \mathrm{~mol} \mathrm{l}^{-1}} \\
{[\mathrm{NaCl}]=4 \times 10^{-2} \mathrm{~mol} \mathrm{l}^{-1}} \\
\mathrm{pH}=3,8\end{array}$ & $99,5 \pm 1,0$ & $0,5 \pm 0,1$ \\
\hline $\begin{array}{c}{\left[^{233} \mathrm{U}\right]=10^{-9} \mathrm{~mol} \mathrm{l}^{-1}} \\
{\left[{ }^{239} \mathrm{Pu}\right]=10^{-9} \mathrm{~mol} \mathrm{l}^{-1}} \\
{[\mathrm{NaCl}]=4 \times 10^{-2} \mathrm{~mol} \mathrm{l}^{-1}} \\
\mathrm{pH}=3,9\end{array}$ & $95,0 \pm 4,0$ & $0,5 \pm 0,1$ \\
\hline
\end{tabular}




\subsection{Essais sur l'urine}

Un concentration connue en ${ }^{233} \mathrm{U}\left(11 \mathrm{~Bq} \mathrm{ml}^{-1}\right)$ a été introduit dans une urine vierge, préalablement filtrée et acidifiée à $\mathrm{pH}=3$, puis divisée en quatre fractions :

- la première fraction d'urine a été mise au contact d'un volume égal de phase organique contenant le calixarène à une concentration de $10^{-3} \mathrm{~mol} \mathrm{l}^{-1}$. Seulement un très faible pourcentage d'uranium est extrait $\left(\operatorname{Ex}_{\mathrm{U}} \approx 6 \%\right)$ dans ces conditions.

- la seconde fraction d'urine a été filtrée sur charbon actif afin d'éliminer une partie de la matière organique. Le filtrat est mis en contact avec un volume égal phase organique dans les mêmes conditions que l'essai précédent. Dans ce cas, $25 \%$ de l'uranium est extrait. Cependant, l'utilisation du charbon actif n'a pas été retenue car une importante quantité d'uranium reste fixée à sa surface.

- ces deux premiers résultats montrent que l'élimination de la matière organique présente dans l'urine semble nécessaire avant l'étape d'extraction par le calixarène. La troisième fraction a donc été minéralisée, suivant le protocole radiochimique classique (Harduin et al., 1996). L'étape finale de ce protocole conduit à l'obtention d'un résidu solide qui doit être dissout dans l'acide nitrique concentré. Sachant que l'extraction optimale de l'uranium par le calixarène requière un $\mathrm{pH}$ en phase aqueuse supérieur ou égal à 3,8 , l'ajustement du $\mathrm{pH}$ à cette valeur est nécessaire. Toutefois, cette augmentation de $\mathrm{pH}$ entraîne la formation d'hydroxydes insolubles. Ce protocole de minéralisation n'est donc pas approprié à l'utilisation du calixarène, la distribution de l'uranium entre les phases aqueuse et organique pouvant varier suivant la sorption de l'uranium sur le précipité.

- pour la dernière fraction, une méthode de minéralisation a été développée avec le peroxyde d'hydrogène (Sansoni et Kracke, 1968). Le résidu est dissout dans une solution d'acide nitrique diluée, puis le $\mathrm{pH}$ est ajusté à 3,8 avec de l'ammoniaque. La solution obtenue est mise en contact avec la phase organique. Dans ces conditions, l'uranium est extrait à $97 \pm 2 \%$.

\section{Discussion}

Cette étude préliminaire a permis de confirmer les hypothèses émises concernant le choix du calixarène utilisé pour l'extraction sélective de l'uranium dans l'urine. Un mécanisme d'extraction de l'uranium par le calix[6]arène-triacide sélectionné a été proposé pour des solutions synthétiques de composition connue. L'efficacité du calixarène a été mise en évidence par la courbe de variation de l'extraction en fonction du $\mathrm{pH}$. Cette courbe fait apparaître un palier correspondant à $100 \%$ d'extraction de l'uranium à partir de $\mathrm{pH}=3,8$. 
La sélectivité du calix[6]arène-triacide pour l'uranium a été étudiée vis-àvis du sodium, qui est un des composants majoritaires de l'urine (Ciba-Geigy, 1981), et vis-à-vis du plutonium, qui est un autre radioélément mesuré en routine en raison de sa forte radiotoxicité. L'étude a montré que quelles que soient les conditions, le sodium n'est jamais extrait par le calix[6]arène-triacide. Ce calixarène ne permet pas au cation alcalin, sphérique, de s'insérer dans sa cavité et ne joue pas non plus le rôle d'un extractant acide vis-à-vis de ce cation. D'après le diagramme de répartition des espèces du plutonium à la valence IV, à $\mathrm{pH}$ avoisinant 4 , plusieurs espèces hydrolysées cohabitent en solution. Les études réalisées en considérant différentes concentrations en plutonium introduites en phase aqueuse ont montré que le plutonium n'est jamais extrait et n'entraîne pas de diminution de l'extraction de l'uranium.

L'efficacité d'extraction du calix[6]arène-triacide vis-à-vis de l'uranium a aussi été démontrée sur une urine réelle. La technique couramment appliquée (Harduin et al, 1996) dans les laboratoires d'analyse et biologie médicale consiste, après précipitation des actinides et minéralisation, à effectuer une chromatographie sur colonnes à l'aide de résines échangeuses d'ions de la société EICHROM. Concernant l'utilisation des calixarènes, seule une adaptation du procédé de destruction de la matière organique a été nécessaire pour permettre l'extraction quantitative de l'uranium présent en solution. Cependant, ce résultat a été obtenu en utilisant le benzène comme solvant du calixarène. Les mécanismes d'extraction sont dépendants des solvants utilisés en phase organique. Les résultats de cette étude le confirme car, en présence de dichlorométhane, le sodium se trouvait coextrait avec l'uranium alors que l'utilisation du benzène permet de s'affranchir de cette coextraction. Toutefois, le caractère toxique de ce solvant interdit l'application de cette méthode d'extraction par les calixarènes, le benzène doit donc être remplacé absolument. Des recherches sont en cours afin de trouver un autre solvant permettant de solubiliser le calixarène et d'extraire sélectivement l'uranium.

Ces premières études confirment l'orientation des recherches, vers les macrocycles de la famille des calixarènes, prise afin d'améliorer la sélectivité de l'extraction des actinides à l'état de traces dans des matrices biologiques de type urine. Il a été possible de construire une molécule dont la taille et la nature des fonctions greffées sont particulièrement bien adaptées à l'extraction sélective de l'uranium. Cette technique permettant de séparer l'uranium du plutonium a l'avantage de donner des résultats plus reproductibles que la technique existante, quand ces deux éléments sont à très basse concentration dans l'urine. D'autres études sont en cours concernant la sélectivité de ce calixarène pour l'uranium vis-à-vis d'autres cations. Les cations divalents seront particulièrement pris en compte car le mécanisme d'extraction met en évidence l'échange de deux protons contre l'ion $\mathrm{UO}_{2}^{2+}$. Par ailleurs les cations trivalents seront aussi étudiés car le calixarène dispose de trois protons mobiles. 


\section{Conclusion}

Le calix [6]arène-triacide, 46 a montré une forte affinité pour l'uranium car il présente une structure adéquate à la structure de coordination de l'ion uranyle. Ces résultats sont encourageants quant à la séparation sélective de l'uranium dans des échantillons d'urine.

Quelle que soit la composition de la phase aqueuse, le pourcentage d'extraction de l'uranium obtenu a toujours été supérieur à $95 \%$. Sur des solutions synthétiques, les valeurs optimales des paramètres d'extraction (concentrations, $\mathrm{pH}$ ) ont été déterminées et un mécanisme d'extraction a pu être proposé. La sélectivité du calixarène choisi, pour l'uranium, a été démontrée vis-à-vis du sodium et du plutonium.

Sur des échantillons d'urine, après une adaptation de l'étape de minéralisation aux conditions d'utilisation du calixarène, la totalité de l'uranium a été extraite par la phase organique.

Remerciements. Les auteurs tiennent à remercier Mlles C. Brugère, C. Dousse et M.P. Ouallet qui nous ont aidé à réaliser les analyses.

\section{RÉFÉRENCES}

Alexander V. (1995) Design and synthesis of macrocyclic ligands and their complexes of lanthanides and actinides. Chem. Rev., 95, 273-342

Araki K., Hashimoto N., Otsuka H., Nagasaki T. et Shinkai S. (1993) Molecular design of a calix[6]arene-based super uranophile with $\mathrm{C}_{3}$ symetry. High $\mathrm{UO}_{2}^{2+}$ selectivity in solvent extraction. Chem. Lett., Vol, 829-832

Baglan N., Dinse C., Cossonnet C., Abidi R., Asfari Z., Leroy M. et Vicens J. Investigation of U(VI) extraction with calixarene. Application to analysis of urine sample. In : Proc. 6th Int. Conf.on Low level measurements of actinides and long-lived radionuclides in biological and environmental samples. La Hague, September 1996. N.P. Singh et J.C. Harduin, Eds., J. Radioanal. Nucl. Chem. (to be published).

Casnati A., Minari P., Pochini A. et Ungaro R. (1991) Conformational freezing of p-tert-butylcalix[6]arene in the cone structure by selective functionalization at the lower rim : synthesis of a new preorganized ligand. I. Chem. Soc., Chem. Commun., Vol, 1413-1414.

Ciba-Geigy (1981) Units of measurement, body fluids, composition of the body, nutrition. Geigy Scientific Tables. Ciba-Geigy limited: Bale.

Grenthe I., Fuger J., Konings R.J.M., Lemire R.J., Muller A.B., N'guyen-Tung C. et Wanner H. (1992) Chemical Thermodynamics of uranium. North Holland: Amsterdam.

Gutsche C.D. (1989) Calixarenes. Royal Society of chemistry. Thomas Graham House. Science Park : Cambridge CB4 4WF.

Harduin J.C., Peleau B. et Levavasseur D. (1996) Détermination analytique des actinides dans les milieux biologiques. Radioprotection, 31 (2), 229.

Dietrich B., Viout P. et Lehn J.M. (1991) Aspects de la chimie des composés macrocycliques, Inter Editions /Editions du CNRS - Paris Meudon. 
Montavon G. (1996) Extraction liquide-liquide des ions U(VI), Np(V), et Th(IV) par deux calix(4)arènes carboxyles, et effet des ions alcalins $\mathrm{Na}^{+}$et $\mathrm{K}^{+}$, Thèse, Université Paris XI.

Nagasaki T. et Shinkai S. (1991) Synthesis and solvent extraction studies of novel calixarene-based uranophile bearing hydroxamic groups. J. Chem. Soc. Perkin Trans., 2, 1063-1066.

Otsuka H., Araki K. et Shinkai S. (1995) Syntheses of all possible calix[6]arene derivatives with MeOand $\mathrm{ROCOCH}_{2} \mathrm{O}$ - substituents and their metal binding properties. Tetrahedron, 51 (32), 8757 8770 .

Sansoni B. et Kracke W. (1968) Aufschluss und Veraschung organischer Substanzen durch Radikale in wässriger Lösung. Z. Anal. Chem., 209.

Shinkai S., Koreishi H., Ueda K., Arimura T. et Manabe O. (1986) A new hexacarboxylate uranophile derived from calix[6]arene. J. Chem. Soc., Chem. Commun., Vol., 233-234.

Shinkai S., Koreishi H., Ueda K., Arimura T. et Manabe O. (1987) Molecular design of calixarenebased uranophile which exhibit remarkably high stability and selectivity. J. Am. Chem . Soc., 109, 6371-6376.

Vicens J. et Böhmer V. (1991) Calixarenes (1991) : a versatile class of macrocyclic compounds. Kluwer Academic Publishers. The Netherlands : Dordrecht 